\title{
Comparison of Decompression and Decompression plus Fusion, for Degenerative Spondylolisthesis Management: Randomized Controlled Trial
} Ali Ahmed Abdel Salam Hussanin

Egyptian Fellowship and Arab Board Trainee, Neurosurgery Department, Nasser Institute Hospital Corresponding author: Ali Ahmed Abdel Salam Hussanin; Email: drloyoo@ hotmail.com; Mobile: (+20)01069903794

\begin{abstract}
Background: Degenerative spondylolisthesis is defined as forward slippage of a vertebra with respect to the underlying vertebra, without rupture of the posterior arc, distinguishing it from lytic spondylolisthesis. It thus usually induces lumbar canal stenosis. There is, however, no strong consensus regarding the various medical and surgical treatments available.

Objective: To assess the long-term clinical results of decompression alone and decompression plus fusion, for degenerative spondylolisthesis.

Patients and methods: We randomly assigned 63 patients who had lumbar spinal stenosis due to degenerative spondylolisthesis at the L4/5 level to undergo either decompression alone (decompression group), decompression plus fusion (fusion group). Three patients refused to undergo randomization; therefore, the remaining 60 patients were randomly assigned.

Results: In total, 60 patients underwent randomization. The follow-up rate at 3 years was $85 \%$. The fusion group showed higher blood loss, longer postoperative hospital stay and a longer operative time than the decompression group. As regard clinical outcomes, all scores significantly improved in the postoperative period and these outcomes were maintained at 3 years postoperatively in each group. There were no significant differences found among the two groups at 1 and 3 years postoperatively.

Conclusion: Decompression plus fusion does not have better results than decompression only in the management of patients with lumber spinal stenosis with low grade degenerative listhesis.
\end{abstract}

Keywords: Decompression, Decompression plus fusion, Degenerative Spondylolisthesis.

\section{INTRODUCTION}

Degenerative spondylolisthesis (DS) is pathological disease referred to forward slippage of the vertebral body that result in symptoms of neurogenic claudication and backache ${ }^{(\mathbf{1})}$.

It mostly occurs in patients older than 50 years being more common in females than in males ${ }^{(2)}$. A large number of symptomatic patients with spondylolisthesis have Meyerding grade I spondylolisthesis, the classification system is based on the degree of slippage ${ }^{(3)}$.

Conservative management consists of physical therapy, epidural injections, anti-inflammatory agents, and opioid analgesic agents ${ }^{(2)}$. However, indications to proceed from conservative treatment to operative intervention depends on progressive neurological deficit, persistent severe backache and/or leg pain, and bladder or bowel symptoms three months following trial of nonoperative interventions ${ }^{(\mathbf{1})}$.

The first line in management of this condition involves conservative management. Approximately $10 \%-15 \%$ of patients develop an incapacitating backaches and/or leg pain (BP and/or LP), which requires surgery ${ }^{(4)}$.

The aim of surgical treatment is decompression of the spinal canal and dural sac from degenerative bony and ligamentous overgrowth ${ }^{(5)}$. Conventional surgical options include decompression and decompression plus fusion ${ }^{\left({ }^{6}\right.}$. The aim of lumbar decompression is to decompress the neural elements while preserving stability ${ }^{(7)}$.

Aim of the work was to assess the long-term clinical results of decompression alone and decompression plus fusion, for degenerative spondylolisthesis.

\section{PATIENTS AND METHODS}

Study Design: This was a prospective, randomized controlled trial.

\section{Ethical and patient approval:}

Patient selection followed approval and registration of this randomized trial from Nasser Institute Hospital. A signed written informed consent was taken from all involved patients. 63 patients who underwent spinal operation for lumbar spinal stenosis with degenerative spondylolisthesis at the L4/5 level from Neurosurgery Department, Nasser Institute Hospital. Those patients who had lumbar spinal operation, multilevel stenosis, or foramenal stenosis were excluded from the beginning. Doctors explained to every patient the clinical condition and the indication of operative intervention and after patient approval of surgical intervention, every patient was invited to participate in the study. Of 63 patients, three 
patients refused to undergo randomization. Therefore, the remaining 60 patients were randomly assigned to undergo either decompression alone (decompression group) or decompression and posterolateral fusion with autogenous iliac bone graft and pedicle screw fixation (fusion group), in 1:1 allocation by an independent doctor, according to computer-generated random number tables. Of these 60 patients, $57(92.1 \%)$ provided information on outcomes.

The diagnosis of lumbar spinal stenosis depends on the presence of typical symptoms, such as neurogenic claudication and radicular leg pain that is associated with neurological signs in addition to the findings from magnetic resonance imaging scans and/or myelograms of stenosis at L4/5 level. Degenerative spondylosis is defined as the presence of $>3 \mathrm{~mm}$ of spondylolisthesis of the L4 vertebra on a plain lateral radiograph. Dynamic instability is defined as a change of $>10$ degrees of angulation or $>4 \mathrm{~mm}$ of translation of the vertebrae between flexion and extension of the spine ${ }^{(2)}$.

All surgeons participated in the study routinely performed the 3 trial interventions. Preoperative and perioperative patient data like age, sex, duration of operation, amount of blood loss, duration of postoperative hospital stay and operative and postoperative complications were collected.

Clinical Follow-up: the Japanese Orthopaedic Association (JOA) score (the score ranges from -6 to 29 based on 3 subjective symptoms, 3 clinical signs including straight-leg raising, 7 activities of daily living and bladder function) and Visual Analogue Scale (VAS) scores for lower back pain and leg pain (ranging from 0 to 100, with higher scores indicating more severe pain) before starting the operation, at 1 year post- operatively and 3 years postoperatively were collected.
The primary outcome degree of lower back pain as measured using the VAS score for secondary outcomes including the JOA score and the VAS score for leg pain. The JOA score was obtained by the operator. The VAS scores were completed with another doctor who was not involved in the study. As regards the radiographic evaluation, we investigated the degree of progression of slippage at 3 years postoperative. Postoperative slip progression was defined as a change of $>5 \%$ of slip progression compared to the preoperative neutral lateral radiograph. Power analysis on the basis of the results of a previous prospective studies. A power analysis with a significance of 0.05 , power of $80 \%$, an assumed SD of 2.0 and expected change in pain score for the lower back of 1.6 was identified. To calculate the sample size of the stunumber, at least 25 subjects were required for detection a significant change in the VAS score for lower back pain between both groups (the decompression and fusion groups).

\section{Statistical analysis}

Recorded data were analyzed using the statistical package for social sciences, version 20.0 (SPSS Inc., Chicago, Illinois, USA). Quantitative data were expressed as mean \pm standard deviation (SD). Qualitative data were expressed as frequency and percentage. Independent-samples t-test of significance was used when comparing between two means. Chisquare (x2) test of significance was used in order to compare proportions between two qualitative parameters.

The confidence interval was set to $95 \%$ and the margin of error accepted was set to $5 \%$. The p-value was considered significant as the following: $\mathrm{P}$-value $\leq$ 0.05 was considered significant. P-value $<0.001$ was considered as highly significant. P-value $>0.05$ was considered insignificant.

\section{RESULTS}

Table (1): Demographic characteristics of the patients.

\begin{tabular}{|l|c|c|c|}
\hline & $\begin{array}{c}\text { Decompression } \\
\text { Group }(\mathbf{N}=\mathbf{2 9})\end{array}$ & $\begin{array}{c}\text { Fusion Group } \\
\mathbf{( N = 3 1 )}\end{array}$ & P \\
\hline Age (y) & $63.4 \pm 8.6$ & $61.2 \pm 6.7$ & 0.93 \\
\hline Female sex [n (\%)] & $13(42)$ & $22(53)$ & 0.29 \\
\hline Degree of vertebral slip (mm) & $6.5 \pm 2.2$ & $8.1 \pm 3.8$ & 0.98 \\
\hline Dynamic instability [n (\%)] & $13(42)$ & $15(46)$ & $>0.87$ \\
\hline
\end{tabular}

There were no significant differences among the 2 groups in any of the preoperative variables (Table 1). Regarding perioperative variables, mean blood loss was significantly higher and operative time was longer in the fusion group than in the decompression group $(\mathrm{P}<0.001)$. (Table 2$)$. 
Table (2): Surgical complications.

\begin{tabular}{|l|c|c|c|}
\hline & $\begin{array}{c}\text { Decompression Group } \\
(\mathbf{N}=\mathbf{2 9})\end{array}$ & $\begin{array}{c}\text { Fusion Group } \\
(\mathbf{N}=\mathbf{3 1})\end{array}$ & P \\
\hline Estimated blood loss & $110 \pm 25.6$ & $318 \pm 77.4$ & $<0.001^{*}$ \\
\hline Operation time & $118 \pm 28.5$ & $256 \pm 50$ & $<0.001^{*}$ \\
\hline Duration of hospital stay after Surgery & $11.6 \pm 2.5$ & $14.1 \pm 3.6$ & $0.007^{*}$ \\
\hline Postoperative slip progression (\%) & 23.6 & 0 & $0.02^{*}$ \\
\hline Complications & & & \\
Any & 1 & 8 & 0.08 \\
Dural tear & 0 & 2 & \\
Delusion & 0 & 0 & \\
Hematoma & 1 & 0 & \\
Meralgia & 0 & 5 & \\
Pulmonary embolism & 0 & 1 & \\
Misplacement of pedicle screw & 0 & 0 & \\
\hline
\end{tabular}

* Statistically significant.

The duration of postoperative hospital stay was significantly longer in the fusion group than in the decompression group $(\mathrm{P}=0.005)$ (Table 2$)$.

Concerning intraoperative and perioperative complications, dural tears occurred in 2 patients in the fusion group. Meralgia paresthetica due to compression of the lateral femoral cutaneous nerve occurred in 5 patients in the fusion group. A postoperative symptomatic hematoma that required bed rest, but not reoperation, occurred in 1 patient in the decompression group. Pulmonary embolism occurred in 1 patient in the fusion group (Table 2).

Table (3): Showing patients deposition in both groups.

\begin{tabular}{|l|c|c|}
\hline & Group I Decompression only & Group II decompression plus fusion \\
\hline Number of enrolled patients & 29 & 31 \\
\hline Follow up at 1 year & $28(96.5 \%)$ & $30(96.71)$ \\
\hline Follow up at 3 year & $23(79.3 \%)$ & $28(90.3 \%)$ \\
\hline
\end{tabular}

Follow-up rates at 1 and 3 years postoperatively were $96.5 \%$ and $79.3 \%$ in the decompression group and 96.7 and $90.3 \%$ in the fusion group respectively (Table 3). During the follow-up period, revision of the operation was performed in 1 patient in the fusion group because of nonunion of the fused segments. As regards radiographic findings, postoperative slip progression was significantly higher in the decompression group than in the fusion group $(\mathrm{P}=0.02,0.02$, respectively). Interestingly, preoperative dynamic instability was not associated with postoperative slip progression in this study $(\mathrm{P}>0.99)$. Regarding clinical outcome, all scores improved postoperatively in both groups. Moreover, all outcome measures showed no statistical differences between the two groups at 1 and 3 years postoperatively.

\section{DISCUSSION}

In our study, although decompression alone and decompression plus fusion did not result in any statistical differences as regards the subjective and patient-based, outcomes at 1 and 3 years postoperatively. The amount of blood loss and duration of operation were less and shorter in the decompression group.

Many spinal surgeons consider degenerative spondylolisthesis with a $3 \mathrm{~mm}$ translation a sign of instability leading to some facilities to perform instrumentation operation for all cases with degenerative spondylolisthesis. Actually, decompression with fusion surgery has become the standard treatment for degenerative spondylolisthesis and in the United States, over $90 \%$ of patients with degenerative spondylolisthesis undergoing surgery now undergo a decompression with fusion, regardless the severity of the spondylolisthesis ${ }^{(9)}$. However, while the use of pedicle screws may lead to a higher fusion rate, it did not always lead to improvements in degree of pain in the back or the lower limbs ${ }^{(\mathbf{1})}$.

$$
\text { investigation, Forsth et al. (10) }
$$

instrumentation of the slipped spine showed no superior outcome compared to decompression only as regard the VAS scores for lower back pain and leg pain and the JOA score are in line with our study. These results may again raise the question of whether local spinal instability due to degenerative spondylolisthesis causes lower back pain and/or lower quality of life. Actually, a prospective comparative studies showed no statistical differences between decompression with fusion and decompression as regards measuring the JOA back pain evaluation questionnaire that scores pain-related disorders, lumbar spine dysfunction, walking ability, social life dysfunction, and psychological disorders ${ }^{(\mathbf{1 1})}$. Moreover, decompression only without fusion for stable spondylolisthesis 
patients with leg-dominant pain is significantly less in cost than instrumented fusion ${ }^{(\mathbf{1 2})}$.

It was found that, the results of our study are partially inconsistent with the results of a previous randomized comparative studies of Ghogawala $\boldsymbol{e t}$ al. (13).

Other study revealed that the addition of lumbar spinal fusion to laminectomy was associated with a greater increase in SF-36 physical component summary scores at 2 and 4 years postoperatively ${ }^{(\mathbf{1 4})}$. However, results of our study did not reveal this association in the JOA scores. Although both studies utilized different evaluation tools as an outcome measure, the JOA score was significantly correlated with the subscales of the SF-36, especially physical functioning. This difference may result from the operative procedures and reoperation rate between the different studies. Although in a study that was done by Ghogawala et al. (13) preforming complete laminectomy with partial removal of the medial facet joint. In our study wide fenestration was performed, a procedure in which only the medial parts of the inferior facets and the adjoining ligamentum flavum are removed ${ }^{(\mathbf{1 5})}$ for decompression to lessen the damage of the facet joint and preserve the spinous process.

Thus, the incidence of postoperative instability caused by the decompression procedure occurred much less in our study. In addition, the study done by Ghogawala et al. ${ }^{(13)}$ had a higher reoperation rate during the follow- up period in the decompression group than in the fusion group ( $34 \%$ vs. $14 \%$ respectively) that is in contrast to our study, which showed overall low reoperation rates $(0 \%$, and $3 \%$, respectively) as reoperation is a risk factor for poor surgical results ${ }^{(\mathbf{1 3})}$.

In our study, mean amount of blood loss was significantly higher in fusion group and operative time was longer than in the decompression group. These results are consistent with that of Koenig $\boldsymbol{e t}$ al. ${ }^{(1)}$ study. Many studies have found that prolonged operative time and blood loss may be due to higher intraoperative and postoperative complications.

In addition, most spinal surgeons found that patients who underwent laminectomy only required less blood transfusions compared to those who underwent instrumentation operation ${ }^{(\mathbf{1})}$.

In our study, the 3 patients who required autologous blood transfusions were in the decompression plus fusion group. Additional instrumentation causes higher blood loss, that lead to the higher incidence of blood transfusions. In addition to that blood transfusion is associated with its risk of complications, such as infection, reaction and anaphylactic shock (16). Even autologous blood transfusion has its risk of bacterial contamination and hemolysis that result in massive blood loss ${ }^{(17)}$.

Also, there were no significant difference as regards the duration of postoperative hospital stay, which was longer in the fusion group compared to the decompression group. Surprisingly, the overall duration of postoperative hospital stay was longer in this study compared with previous reports ${ }^{(13)}$.

The strength in our study is the usage of original surgical reports, discharge summaries and outpatient medical records to extract accurate information. Also, we only selected patients with 1 level lumbar canal stenosis due to degenerative spondylosis at the L4/5 level to accurately compare the effects of surgical interventions. A second strength is the predefined analysis plan using validated subjective and patient recorded outcome measures to evaluate neurological recovery and pain ${ }^{(\mathbf{1 8})}$.

\section{CONCLUSION}

Decompression plus fusion does not have better results than decompression alone in the management of patients with lumber spinal stenosis with low grade degenerative listhesis.

Funding: no fund

Conflict of interest: authors have no conflict of interest.

\section{REFERENCE}

1. Koenig S, Jauregui JJ, Shasti M et al. (2019): Decompression versus Fusion for Grade I Degenerative Spondylolisthesis: A Meta-Analysis, Global Spine Journal, 9 (2): 155-161.

2. Weinstein JN, Lurie JD, Tosteson TD et al. (2007): Surgical versus non- surgical treatment for lumbar degenerative spondylolisthesis. N Engl J Med., 356: 2257-2270.

3. Jacobsen S, Sonne-Holm S, Rovsing H et al. (2007): Degenerative lumbar spondylolisthesis: an epidemiological perspective: the Copenhagen Osteoarthritis Study. Spine, 32: 120-125.

4. Kelleher MO, Timlin M, Persaud O et al. (2010): Success and failure of minimally invasive decompression for focal lumbar spinal stenosis in patients with and without deformity. Spine, 35: 981-987.

5. Ulrich NH, Burgstaller JM, Pichierri G et al. (2017): Decompression surgery alone versus decompression plus fusion in symptomatic lumbar spinal stenosis: a Swiss prospective multicenter cohort study with 3 years of follow-up. Spine, 17: 164.

6. Donnarumma P, Tarantino R, Nigro L et al. (2016): Decompression versus decompression and fusion for degenerative lumbar stenosis: analysis of the factors influencing the outcome of back pain and disability. J Spine Surg., 2: 52-58.

7. Moisi M, Fisahn C, Tkachenko L et al. (2016): Unilateral laminotomy with bilateral spinal canal decompression for lumbar stenosis: a technical note. Cureus., 8: 623-266.

8. Inose H, Yamada T, Mulati M et al. (2018): Bone turnover markers as a new predicting factor for non-union after spinal fusion surgery. Spine, 43: 29-34.

9. Kepler CK, Vaccaro AR, Hilibrand AS et al. (2014): National trends in the use of fusion techniques to treat degenerative spondylolisthesis. Spine, 39: 1584-1589. 
10. Forsth P, Olafsson G, Carlsson T et al. (2016): A randomized, controlled trial of fusion surgery for lumbar spinal stenosis. N Engl J Med., 374: 1413-1423.

11.Aihara T, Toyone T, Murata $Y$ et al. (2018): Degenerative lumbar spondylolisthesis with spinal stenosis: a comparative study of 5-year outcomes following decompression with fusion and microendoscopic decompression. Asian Spine J., 12: 132139.

12. Kim S, Mortaz Hedjri S, Coyte PC et al. (2012): Costutility of lumbar decompression with or without fusion for patients with symptomatic degenerative lumbar spondylolisthesis. Spine J., 12: 44-54.

13. Ghogawala Z, Dziura J, Butler WE et al. (2016): Laminectomy plus fusion versus laminectomy alone for lumbar spondylolisthesis. N Engl J Med., 374: 14241434.

14. Fujiwara A, Kobayashi N, Saiki K et al. (2003): Association of the Japanese Orthopaedic Association score with the Oswestry Disability Index, Roland-Morris Disability Questionnaire, and short-form 36. Spine, 28: 1601-1607.

15. Nakai O, Ookawa A, Yamaura I (1991): Long-term roentgenographic and functional changes in patients who were treated with wide fenestration for central lumbar stenosis. J Bone Joint Surg Am., 73: 1184-1191.

16. Bihl F, Castelli D, Marincola F et al. (2007): Transfusion-transmitted infections. J Transl Med., 5: 2530.

17. Walunj A, Babb A, Sharpe R (2006): Autologous blood transfusion. Contin Educ Anaesth Crit Care Pain, 6: 192196.

18. Briggs M, Closs JS (1999): A descriptive study of the use of visual analogue scales and verbal rating scales for the assessment of postoperative pain in orthopedic patients. $\mathbf{J}$ Pain Symptom Manage., 18: 438-446. 